\title{
9. THE INFLUENCE OF EXPOSURE DURATION AND \\ TRAIL ORIENTATION ON \\ PHOTOGRAPHIC METEOR SPECTRA
}

\author{
I. Halliday \\ (Dominion Observatory, Ottawa, Canada)
}

\begin{abstract}
A BSTRACT
The effects on photographic meteor spectra produced by an occulting shutter and by the geometric orientation of the trail with respect to the camera are considered. A Perseid meteor photographed with both an occulted and unocculted spectrograph shows that large changes in the relative intensity of spectral lines can be caused by a shutter. The strong wake spectrum of this meteor is studied and a classification of multiplets according to duration and wake intensity is presented. It is emphazised that a strong wake is produced by a coasting gas rather than by particles or droplets. The gas may coast an appreciable distance during the radiating lifetime of some multiplets.

The spectrum of a bright Quadrantid meteor is used to show that long-duration multiplets are strongly enhanced when the orientation of the trail lengthens the effective path length of the wake column, i.e., the meteor is observed near its radiant. The abundance of calcium appears to be above average in this meteor.

Multiple photographs of the $\lambda 5577$ line of neutral oxygen have been secured showing a decay time in excess of $1.5 \mathrm{sec}$. The decay is more rapid at lower heights and it appears that the mechanism of excitation to the metastable level operates for an interval of about $0.1 \mathrm{sec}$.

As more accurate photometry of meteor spectra is achieved it will be essential to give careful attention to all of these effects.
\end{abstract}

\section{Introduction}

Photographic meteor spectra are time exposures of a moving, variable, luminous phenomenon, observed at a particular angle to the axis of symmetry of the meteoric column. These observational conditions introduce complications in the study of meteor spectra, three of which are considered in this paper.

\section{Short-Duration Meteor Wakes}

For bright meteors the luminosity from a particular point on the trajectory may persist for times ranging from a millisecond or less to several seconds. The term meteor wake is used (Millman, 1961) to refer to phenomena of short duration, usually much less than $1 \mathrm{sec}$, originating behind the moving meteoroid. At meteoric velocities a duration of even 5 millisec may correspond to some hundreds of metres of travel of the meteor. When this is combined with the appreciable duration of

Kresák and Millman (eds.), Physics and Dynamics of Meteors, 91-104. C I.A.U. 
luminosity at a single point it is seen that there is no simple relationship between good time resolution and spatial resolution on a meteor photograph. The image of any point on the trail is normally a time exposure for all, or part, of the duration of luminosity at that point. To obtain details of the physical processes involved, a reconstruction of the time variation of the luminosity is required.

Since different emission lines have quite different radiating lifetimes it is essential to employ spectra for such studies. Furthermore, an adequate dispersion is required to minimize uncertainties due to blended spectral features. Early studies of wake spectra have been published by Millman (1950, 1956), who emphasized the low excitation of the spectra, and by Halliday (1958a), who concluded that the wake spectra of bright meteors were due primarily to a coasting cloud of meteoric gas rather than to trailing particles.

Significant data on meteor wakes may be obtained from the study of spectrograms of a bright Perseid meteor photographed at the Meanook and Newbrook Meteor Observatories, Alberta, Canada, at $8^{\mathrm{h}} 15^{\mathrm{m}} 30^{\mathrm{s}} \mathrm{UT}$, August 12, 1963. Much of the Meanook spectrogram is reproduced in Figure 1. This was photographed with a converted K19 aerial camera (called camera A), focal length $305 \mathrm{~mm}$, using a 300 line-per-mm transmission diffraction grating. A rotating shutter with a closed-to-open ratio of $2: 1$ chopped the trail $11 \cdot 3$ times per second.

Three spectrographs at Newbrook also recorded at least part of the spectrum. These were F24 cameras, focal length $203 \mathrm{~mm}$, using 400 line-per-mm gratings. None of these cameras had a rotating shutter. The first-order spectrum from camera $U$ is reproduced in Figure 2. This spectrogram has good focus in the blue but only fair focus at longer wavelengths. It also recorded strong second- and third-order spectra of the blue-violet region with superb definition in the $3800-4000 \AA$ region, where numerous strong multiplets are resolved exceptionally well.

Figures 1 and 2 not only provide material for a comparison of an occulted and unocculted spectrogram of the same meteor, but the strong flares common in the later stages of bright, fast meteors were almost ideally phased with respect to the occulting shutter on camera $\mathrm{A}$ at Meanook. Eight segments of meteor trail were recorded by camera $A$, of which the last five are shown in Figure 1. Heights of various points (above sea level) were computed and are shown in Table 1 for the points designated by letters in Figures 1 and 2. The heights are normal for a bright Perseid meteor. In addition to the values in Table 1, which were based mainly on zero-order images, an exceedingly faint image of the auroral-green line ( $\lambda$ 5577) may be detected on the original plate from camera A between heights of about 116 and $109 \mathrm{~km}$.

Other values pertinent to this meteor are: elevation of the Perseid radiant, 49.4; elevation of segment 8 (camera A), 24.6; elongation of segment 8 from radiant, $78^{\circ} .9$; duration of meteor $0.67 \mathrm{sec}$.

A comparison of the two figures in the region of the flares shows the following relationships: A substantial flare began near the end of segment 7 (camera A) and 


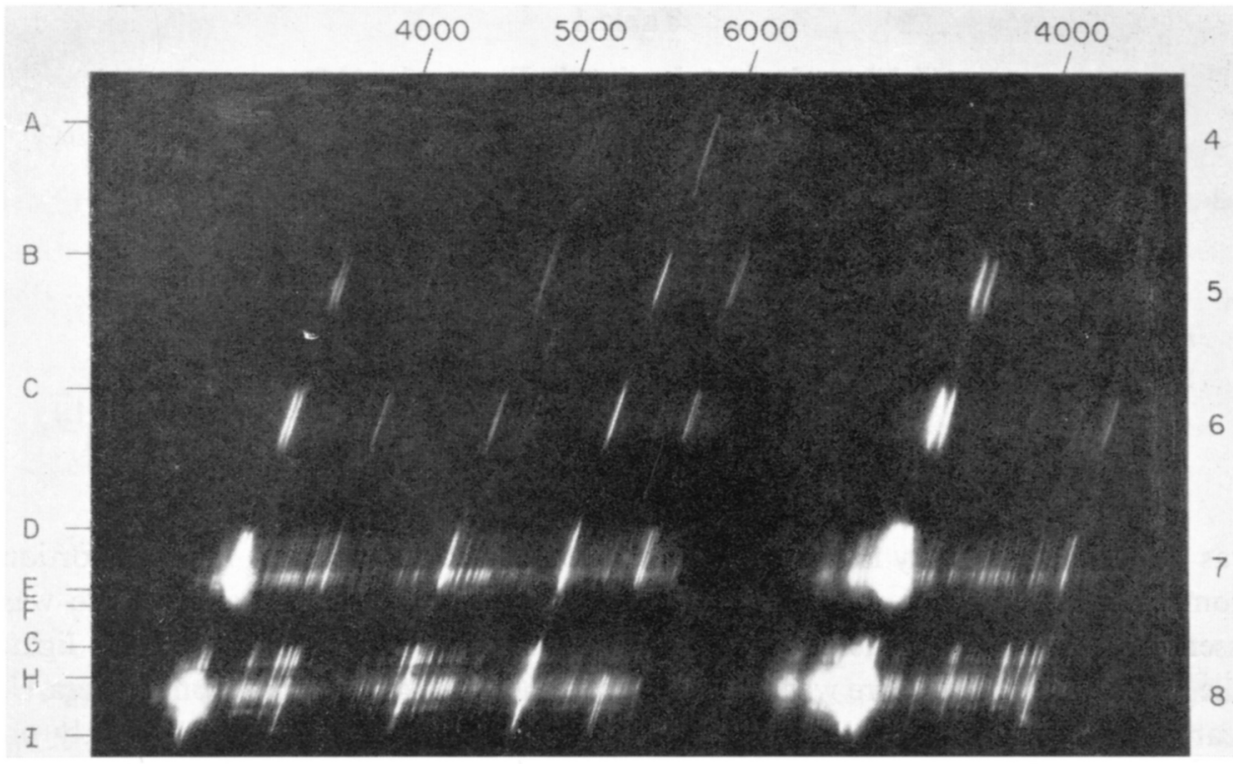

FIG. 1. Perseid meteor spectrum photographed at Meanook with camera A, showing first-order and blue portion of second-order spectrum. Segment numbers appear at right, while letters identify specific points mentioned in text and Table 1.

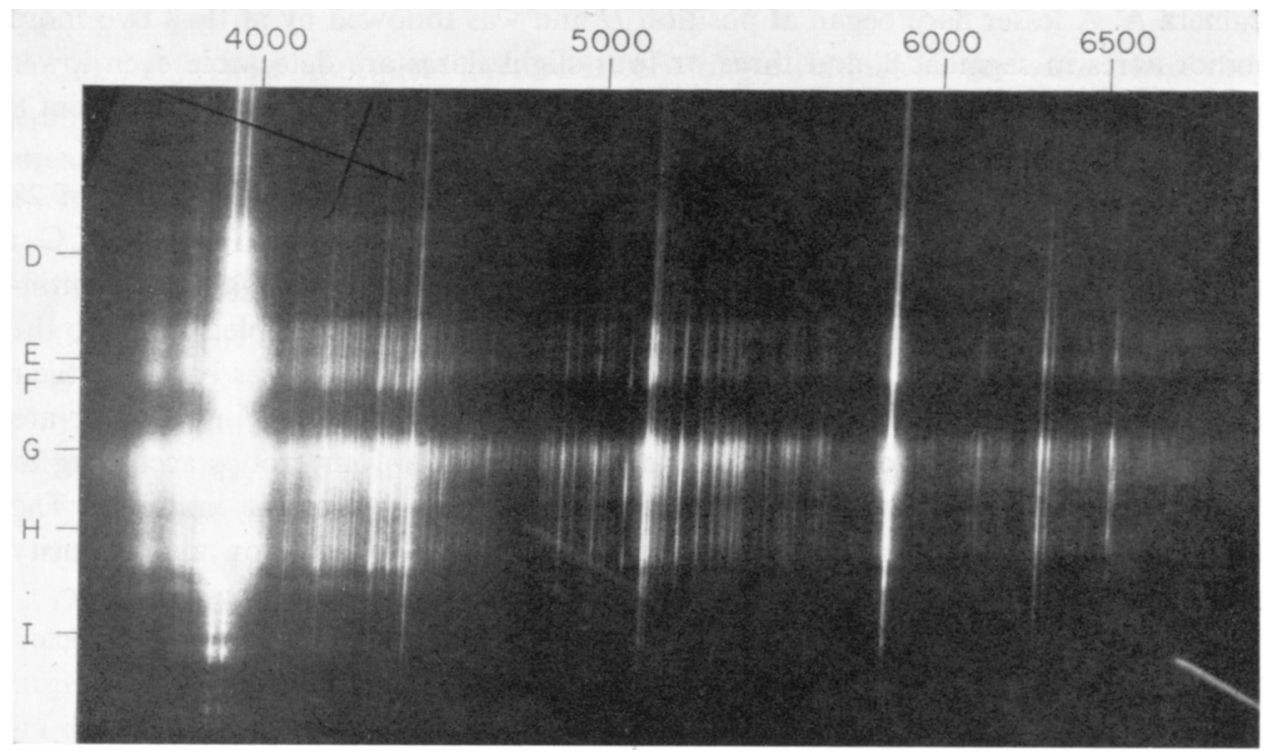

Fig. 2. The first-order spectrum of the same meteor as in Figure 1, photographed with camera $U$ at Newbrook without a rotating shutter. 
Table 1

Heights of selected points in Figures 1 and 2

$\begin{array}{cccc}\text { Location } & \text { Height }(\mathrm{km}) & \text { Location } & \text { Height }(\mathrm{km}) \\ \text { Top } & 110.5 & F & 84.9 \\ \text { A } & 98.5 & G & 83.9 \\ B & 94.5 & H & 82.4 \\ C & 90.4 & I & 81.1 \\ D & 86.4 & J & 79.8 \\ E & 85.1 & & \end{array}$

$A, B, C, D$ and $H$ are the beginning points of segments on camera A. $J$ is the bottom of the trail as observed on camera $U$.

was at a minor intensity minimum as the shutter closed at position $E$. This portion from camera $A$ is thus relatively pure meteor-head radiation since the wake was essentially excluded by the shutter, while camera $U$ recorded the integrated light. The second half of this flare was very similar to the first half, judging from camera $U$, near position $F$. The wake radiation was sufficiently intense to record weakly on camera $\mathrm{A}$, position $F$, after the shutter reopened at $H$, yielding a rather old wake spectrum. The most intense flare occurred near position $G$ while camera A was occulted and produced the strong, detailed and relatively fresh wake spectrum observed between $G$ and $H$. This flare subsided considerably before position $H$, producing the apparent division between the strong wake spectrum and segment 8 on camera A. A lesser flare began at position $H$ and was followed by at least two more minor flares in segment 8 , and three or four slight flares are detectable even lower on camera $\mathrm{U}$. These were not strong enough to record any wake beyond segment 8 on camera A.

Analysis of the wake spectrum near position $G$ leads to the identification of 28 multiplets in this portion of the wake. Of these, seven multiplets of $\mathrm{FeI}$, one of $\mathrm{Ca} I$ and one of $\mathrm{CrI}$ appear to be new additions to previous lists of wake-spectra identifications (Millman, 1956; Halliday, 1963). Table 2 lists these 28 multiplets and also the one metastable multiplet. The table divides multiplets into five classes based on their duration in the luminous meteor phenomenon. Multiplets observed in the wake are confined to the last four classes and these are divided into four groups according to the intensity, in the meteor wake, of the strongest members of the multiplet. The table is thus a two-dimensional classification according to duration and intensity where the duration classes and intensity groups are defined as follows:

Class I: These are multiplets which decay so quickly that they are not detected at all in meteor wakes. Examples include $\mathrm{HI}, 1$, all multiplets of NiI, OII, MgII,

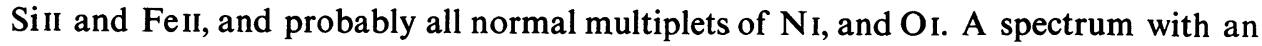
occulted flare such as at position $G$, Figure 1, but recorded on infrared film, would be useful for checking the multiplets of $\mathrm{N}_{I}$ and $\mathrm{O}_{\mathrm{I}}$. 
Table 2

\section{Classification of multiplets according to duration and wake intensity}

Class

I High-excitation multiplets, not present in wake spectrum $\mathrm{HI}_{\mathrm{I}}, \mathrm{O}_{\mathrm{I}}, \mathrm{N}_{\mathrm{I}}, \mathrm{Mg}_{\mathrm{II}}, \mathrm{Si}$ II

A

II

III $\quad \mathrm{NaI}, 1$

CaII, 1

IV

$\mathrm{V}$
B Intensity in wake spectrum

C D

MgI, 2 CaI, 18

FeI, $41 \quad$ FeI, 20, 21, 37, 39, 42

CaI, 2 CaI, 3

FeI, $5 \quad$ CrI, 2

MnI, 2

FeI, 16

FeI, 1, 2, 3,

MgI, 1

Cal, 1

FeI, 13, 14

$\mathrm{O}$, 3F

Class II: These multiplets may be detected in wake spectra but decay rather quickly and will be much reduced in intensity relative to their unocculted strengths. Multiplet 3 of MgI should probably also appear in this class in Table 2, but was not resolvable in Figure 1 from neighbouring multiplets of FeI.

Class III: The division between classes II and III is somewhat arbitrary. Class III multiplets are generally low-excitation ones, which may be rather prominent contributors to both the primary and wake spectra, such as NaI, 1; CaI, 2; CaII, 1, and $\mathrm{Fe} \mathrm{I}, 4,5$, and 15.

Class IV: These multiplets have relatively long durations in the wake where they contribute some of the strongest lines although they are quite weak in the primary meteor spectrum. Two of the additions to the list of wake multiplets in Table 2 are in this class, Fe I, 13 and 14. All seven multiplets in Class IV are intersystem multiplets with low excitation energies. Two other intersystem multiplets, FeI, 39 and 41, have appreciably higher excitations and are found in Class II.

Class $V$ : The only member of this class is multiplet $3 \mathrm{~F}$ of $\mathrm{OI}$, frequently photographed in meteor spectra and termed a metastable train by Millman (1963).

The wake intensity groups are defined as follows:

Group A: major contributor to wake intensity.

Group B: strong wake lines.

Group $C$ : lines of moderate strength.

Group D: only weak or very weak lines observed. (Note that multiplet $3 \mathrm{~F}$ of $\mathrm{OI}$ belongs to group $\mathrm{D}$ in this spectrum but may often be a much stronger feature.)

The influence of the rotating shutter on the appearance of the spectrum is well shown by a study of the relative intensities of two closely spaced lines of FeI, $\lambda 4376$ of multiplet 2 (a class-IV multiplet) and $\lambda 4383$ of multiplet 41 (a class-II multiplet). The intensities near the meteor head are shown by positions such as $E$ or $I$ in Figure 1 
where the rotating shutter just began to occult the camera. Near position $E$, for example, $\lambda 4383$ is stronger than $\lambda 4376$ by at least two stellar magnitudes, or more than a factor of 6 . Near the top of a segment, somewhat below points $D$ or $H$, this ratio has been cut to about a factor of 2 . This corresponds to the integrated radiation during the first 25 millisec. In a fairly fresh wake, e.g. between $G$ and $H$, the first few milliseconds have been discarded by the shutter and $\lambda 4376$ dominates $\lambda 4383$ by a factor of at least 6 . In a rather old wake, such as at position $F, \lambda 4376$ survives weakly while $\lambda 4383$ is completely absent.

A meteor with a single brief flare photographed through a rotating shutter could thus exhibit intensity ratios of at least $6: 1$ in either sense for these two lines, depending only on the phasing of the shutter. With no shutter, as in Figure 2, the integrated intensities are comparable, with $\lambda 4383$ slightly more intense than $\lambda 4376$ in general.

It can thus no longer be considered an adequate description of a meteor spectrum to record the relative intensities of spectral features without due consideration to the effects caused by the presence or absence of a shutter. Lines from class-I or class-II multiplets are strong only near the meteoroid itself, while class-IV multiplets seem to attain their maximum strength some appreciable distance back in the wake. Without a shutter the only hint of this behaviour comes from the progressively larger shift of the class-III and -IV multiplets down the meteor trail from the point where some flare has occurred. More information can be obtained from the occulted spectra but only if the exposure effects at each point are considered.

Ceplecha (1966) studied the spectrum of a bright iron asteroidal fragment which exhibited a prominent wake in direct photographs. He suggested that the transport of material behind the meteoroid in the wake may be in the solid, liquid and gaseous states but that liquid droplets play an important part. On his model the wake radiation at any time is largely due to gas particles just freshly ablated from the trailing droplets.

Ceplecha's wake and its spectrum are normal except for the absence of certain lines because of the chemical composition. A study of the Perseid spectrum in Figure 1, however, shows that this model of ablation from trailing droplets is unacceptable. Small droplets leaving the meteoroid will decelerate more and more rapidly as their size decreases due to ablation. Consider a meteor with velocity $V_{0}$ and a droplet whose velocity has decreased to $V_{1}$ during the time $t_{1}$ since it left the meteoroid. The droplet trails the meteoroid by a distance

$$
\Delta l=\int_{0}^{t_{1}}\left(V_{0}-V_{1}\right) \mathrm{d} t,
$$

and because of the increasing nature of the retardation

$$
\Delta l<\frac{V_{0}-V_{1}}{2} t_{1}
$$


i.e., the droplet cannot trail the meteoroid by as much as half the distance travelled by the meteoroid in the interval. For wakes with relatively large values of $t_{1}, V_{1}$ will approach the minimum value of about $5 \mathrm{~km} / \mathrm{sec}$ required to still produce appreciable luminosity, and $\Delta l$, the retardation, will not exceed about $30 \%$ of $V_{0} t_{1}$, the distance travelled by the meteoroid.

Now the Perseid spectrum shows a wake spectrum at position $F$, among others, in Figure 1. The unocculted spectra show that two flares occurred near this point, one just before the shutter occulted the camera at position $E$ and one just afterwards. The wake spectrum at $F$ is due to the second flare. It was recorded after the shutter reopened at position $H$, some 53 millisec after the flare itself. Comparison of the occulted and unocculted spectra shows that the wake had drifted at most $560 \mathrm{~m}$ down the trail in this time while the meteor itself travelled $3.2 \mathrm{~km}$. In other words, the retardation was at least $82 \%$ of the meteor's travel, which, as shown above, is quite unacceptable for ablating solid or liquid particles. Ceplecha's iron meteor had a rather smooth light curve, which prevents identification of the exact point where wake material left the meteoroid, while the Perseid had such prominent flares that there can be no doubt that a particular flare released the material later observed to produce a certain portion of the wake. The only way to account for the very rapid deceleration is to have atomic-sized particles very soon after the flare, so that the first few collisions with air particles can produce large changes in velocity. The overall momentum of the meteoric material can maintain sufficient coasting velocity to produce the observed excitation for the duration of the wake.

If the wake were produced by evaporation from droplets it would also be difficult to explain the complete absence of lines such as those from Sill in the wake. A droplet from a Perseid meteor will take some time to decelerate from 60 to $40 \mathrm{~km} / \mathrm{sec}$ and should be capable of producing SilI lines during this interval. Rapid initial deceleration, as expected for a gas, is again a sufficient explanation of this rapid quenching of high-excitation lines.

\section{Aspect of the Trail}

Evidence has been obtained which indicates that the photographic record of a meteor spectrum may be strongly influenced by the aspect or orientation of the trail with respect to the point of observation. As noted above, high-excitation multiplets in class I decay so quickly that they are not detected at all in meteor wakes. For these lines the cloud of emitting atoms may be considered to occupy a small volume in the vicinity of the moving meteoroid. The assumption of a spherical shape for this volume should not be grossly in error.

On the other hand, in class IV there are lines from multiplets such as FeI, 1, 2, 3, which we have seen may reach their maximum luminous intensity at a considerable distance behind the meteor. Lifetimes of $0.050 \mathrm{sec}$ are not unusual for such lines 
which, for a Perseid meteor, corresponds to a radiating column at least $3 \mathrm{~km}$ in length. Such a column is very long compared to its width and may be represented quite well by a cylindrical model.

For the high-excitation lines the path length of radiating atoms is the diameter of the effective sphere and is independent of the aspect of the trail, while for the lowexcitation wake lines the effective path length is longer than the diameter of the cylinder by a factor $\operatorname{cosec} \alpha$ where $\alpha$ is the elongation of the point from the meteor radiant. The wake lines, then, may become strongly enhanced relative to lines of shorter duration when a meteor happens to be photographed close to its radiant.

An apparent example of this phenomenon is shown in Figure 3, which shows the spectrum of a Quadrantid meteor photographed at Newbrook near $1^{\mathrm{h}} 13^{\mathrm{m}} \mathrm{UT}$, January 3, 1964. While the time of appearance is some 29 hours before the predicted shower maximum, there is little doubt that it is a Quadrantid meteor. The trail begins about 9.6 from the assumed position of the radiant and the trail is oriented so that the assumed radiant crosses the projection of the observed trail during the 22-min exposure. Furthermore, the trail is only 4.2 in length, yet it is bright and exhibits at least four main flares, suggesting that the angular velocity was low but the duration was not short.

Table 3 shows the heights derived for seven points along the trail which are also

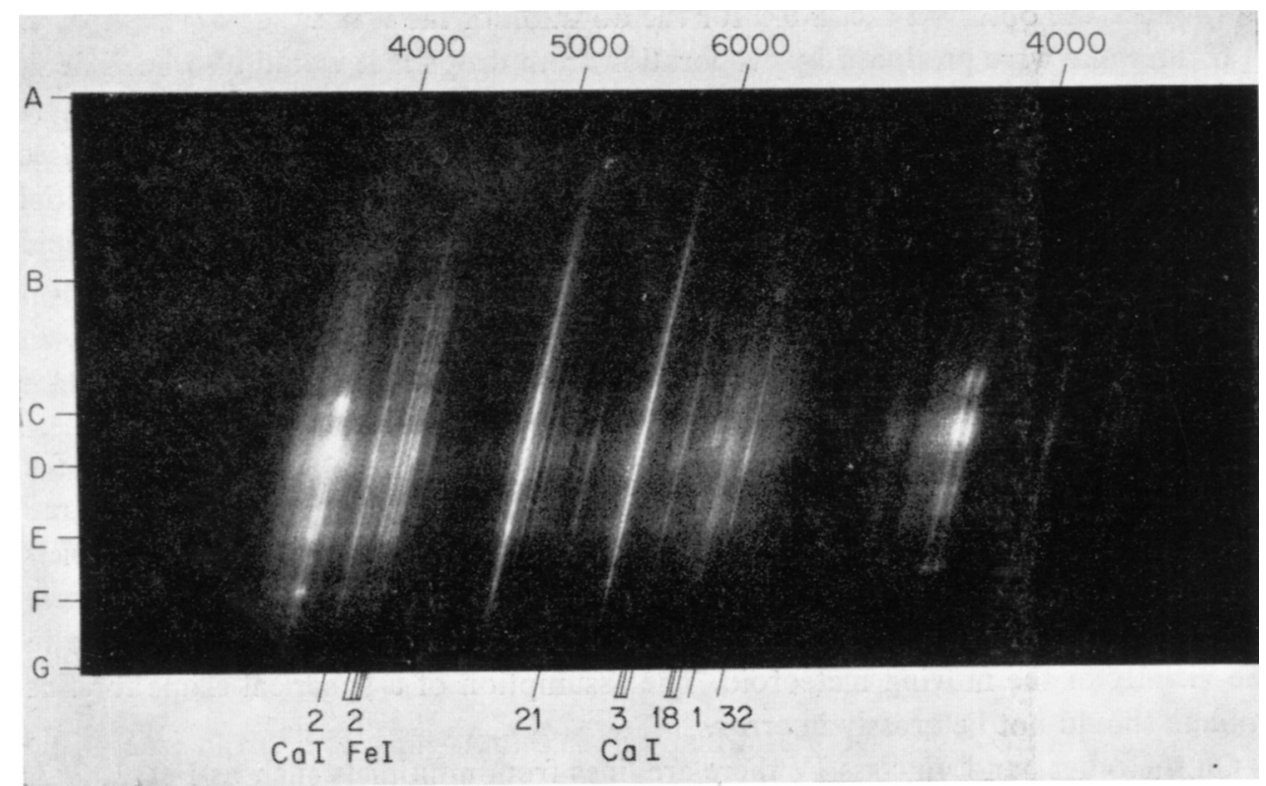

FIG. 3. Spectrum of a Quadrantid meteor photographed near its radiant at Newbrook. Six multiplets of $\mathrm{CaI}$ and one of $\mathrm{FeI}$ are identified at the bottom. Letters at left refer to positions in Table 3. 
Table 3

Heights of points in Quadrantid spectrum (Figure 3)

$\begin{array}{cccc}\text { Point } & \text { Height }(\mathrm{km}) & \text { Point } & \text { Height }(\mathrm{km}) \\ A & 104.5 & E & 78.4 \\ B & 93.7 & F & 76.0 \\ C & 84.5 & G & 72.9 \\ D & 82.0 & & \end{array}$

marked in Figure 3. The heights were computed from the elevation of the radiant combined with a reasonable height for the point of maximum light for a meteor of this velocity $(41 \mathrm{~km} / \mathrm{sec})$. All heights could be in error by some constant factor but are not expected to be in error by more than about $5 \%$.

For the values shown in Table 3 the length of the luminous trail is $86 \mathrm{~km}$, the duration $2 \cdot 1 \mathrm{sec}$, and the angular velocity only 2.0 per second. From a comparison of zero-order image strengths, allowing for the difference in angular velocity between the meteor and star trails, the estimated panchromatic magnitude of this meteor at position $D$, maximum brightness, was about magnitude $-6 \cdot 3$.

The Quadrantid spectrum is typical of a moderately fast meteor in that it exhibits strong lines of neutral atoms together with fairly strong $\mathrm{H}$ and $\mathrm{K}$ lines of Ca II and some contributions from other ions, notably $\mathrm{Si}$ II and $\mathrm{Mg}$ II. The spectrum may be considered somewhat unusual in three respects: (i) the lines of lowest excitation of $\mathrm{Fe}$ I, Mg I, Ca I, Na I, normally associated with meteor-wake radiation, are strongly enhanced in the spectrum relative to other lines from the same elements, (ii) the high-excitation lines from ionized elements appear only in the upper portions of the flares, (iii) lines of Car are unusually prominent in this spectrum.

The explanation for comment (i) appears to be due to the aspect of the trail as seen from Newbrook. The values of $\operatorname{cosec} \alpha$ for the photographed trail decrease from 6.0 at the top of the trail to 4.2 at the bottom, with a value of 4.7 near the point of maximum light. Thus, a definite enhancement of the lines radiated from an effectively cylindrical column should be expected compared with lines from short-lived multiplets. The great strength of Fe I, 2 in this spectrum relative to $\mathrm{Fe} I, 41$ or 43 , is due to this effect. In the Perseid spectrum of Figure 2, $\lambda 4383$ of FeI, 41 is somewhat stronger than $\lambda 4376$, while in the Quadrantid spectrum the presence of multiplet 41 is based on a weak line at $\lambda 4415$ since $\lambda 4383$ is not observed, on this dispersion, due to the unusually strong $\lambda 4376$ of multiplet 2 .

The different light curves observed at the flares for different multiplets, comment (ii) above, are explained by the variations in lifetime combined with the coasting motion of the meteoric gas. The high-excitation class-I lines indicate the instantaneous variation in light curve of the moving meteoroid. (The presence of $\lambda 4481$ of $\mathrm{Mg}_{\text {II }}$ is shown by its effect on the light curve of the blend with $\lambda 4482$ of FeI, 2. Definite 
blobs in the light curve are observed in exact phase with the similar lines of SiII.) For lines with increasing lifetimes the effective mid-position of each flare is shifted further down the trail due to the momentum of the coasting gas cloud, while the line is still being radiated, as was also noted for the Perseid meteor.

The unusual strength of the calcium lines (comment (iii)) is of some interest. Multiplet 1 , at $\lambda 6572$, is the strongest feature in the red region. It is a class-IV line, and although it would be blended with $\mathrm{H} \alpha$ on this dispersion the light curve is typical of the class-IV wake lines and not at all like class I. Multiplets 2 and 3 are quite definite, as would be expected. Three fairly strong lines are attributed to multiplet 18 , one strong blend to multiplet 21 , and multiplet 32 ( $\lambda$ 6717) can be detected. Multiplets $4,5,19,20,22$, and 23 are probable contributors to blends with other features.

The explanation could be either an over-abundance of calcium compounds in this Quadrantid meteor or, possibly, the excitation may be optimum to produce strong lines of CaI without depleting the abundance of Ca I by intense ionization to CaII. Calcium lines seem notably stronger in this Quadrantid than in comparable spectra of Geminid $(36 \mathrm{~km} / \mathrm{sec})$ or Lyrid $(48 \mathrm{~km} / \mathrm{sec})$ meteors which suggests the explanation may be the actual abundance rather than the excitation conditions.

Additional evidence for the influence of the aspect of the trail on meteor spectra is found from the spectrum of a faint Geminid meteor photographed at Newbrook at $10^{\mathrm{h}} 17^{\mathrm{m}} \mathrm{UT}$, December 13, 1963. The fast, low-dispersion spectrograph which secured the spectrum was equipped with a rotating shutter with a closed-to-open ratio of $2: 1$. The spectrum shows about six lines, including a rather prominent auroral green line at $\lambda 5577$ in spite of the loss caused by the shutter. At $36 \mathrm{~km} / \mathrm{sec}$ for a Geminid this appears to be the slowest meteor to exhibit $\lambda 5577$ in its spectrum since previous examples were all meteors with a velocity of at least $48 \mathrm{~km} / \mathrm{sec}$ and generally $60 \mathrm{~km} / \mathrm{sec}$ or larger. The green line is observed for about 0.9 of path length only $9^{\circ}$ from the Geminid radiant, hence this enduring radiation is enhanced by viewing the glowing columm through a path length increased by a factor of $\operatorname{cosec} \alpha$ $=6 \cdot 4$.

It would be of interest to secure spectra of the same meteor from two stations such that there was a large difference in the factor $\operatorname{cosec} \alpha$ for the two spectra. This requires either longer baselines than are normally employed or else a meteor for which $\alpha$ is small at both stations but considerably less at one station than at the other. Such pairs will be rare in collections of meteor spectra. The question of aspect sensitivity is not restricted to meteors, of course. Photographs of Comet ArendRoland (1957) taken between April 23 and 28 showed the sunward spike caused by the increased effective path length of cometary debris in the line of sight while the Earth was passing through the orbital plane of the comet. There was no change in the comet on those days, yet the photographic records are dramatically different because of the location of the observer. 


\section{Long-Duration Meteor Wakes}

The auroral green line of neutral oxygen, $\lambda 5577$, arises from a metastable level and has been studied in meteor spectra by several investigators (Halliday, 1958b, 1960; Millman, 1963; Russell, 1964; Rajchl, 1965). Since spectra photographed through a rotating shutter showed that the decay of the line was measured in tenths of seconds or even more, two spectrographs were designed and constructed to measure the decay rate (Halliday and Griffin, 1963). In these two.instruments the film is rapidly advanced 100 and 150 times per minute (hence they are called cameras 100 and 150) so that consecutive images of the green line are recorded during its decay with exposure times of 0.6 and $0.4 \mathrm{sec}$ for the two instruments.

More than 40 spectra showing multiple images of $\lambda 5577$ have now been secured with these instruments. One of the best is shown in Figure 4, which is the spectrum

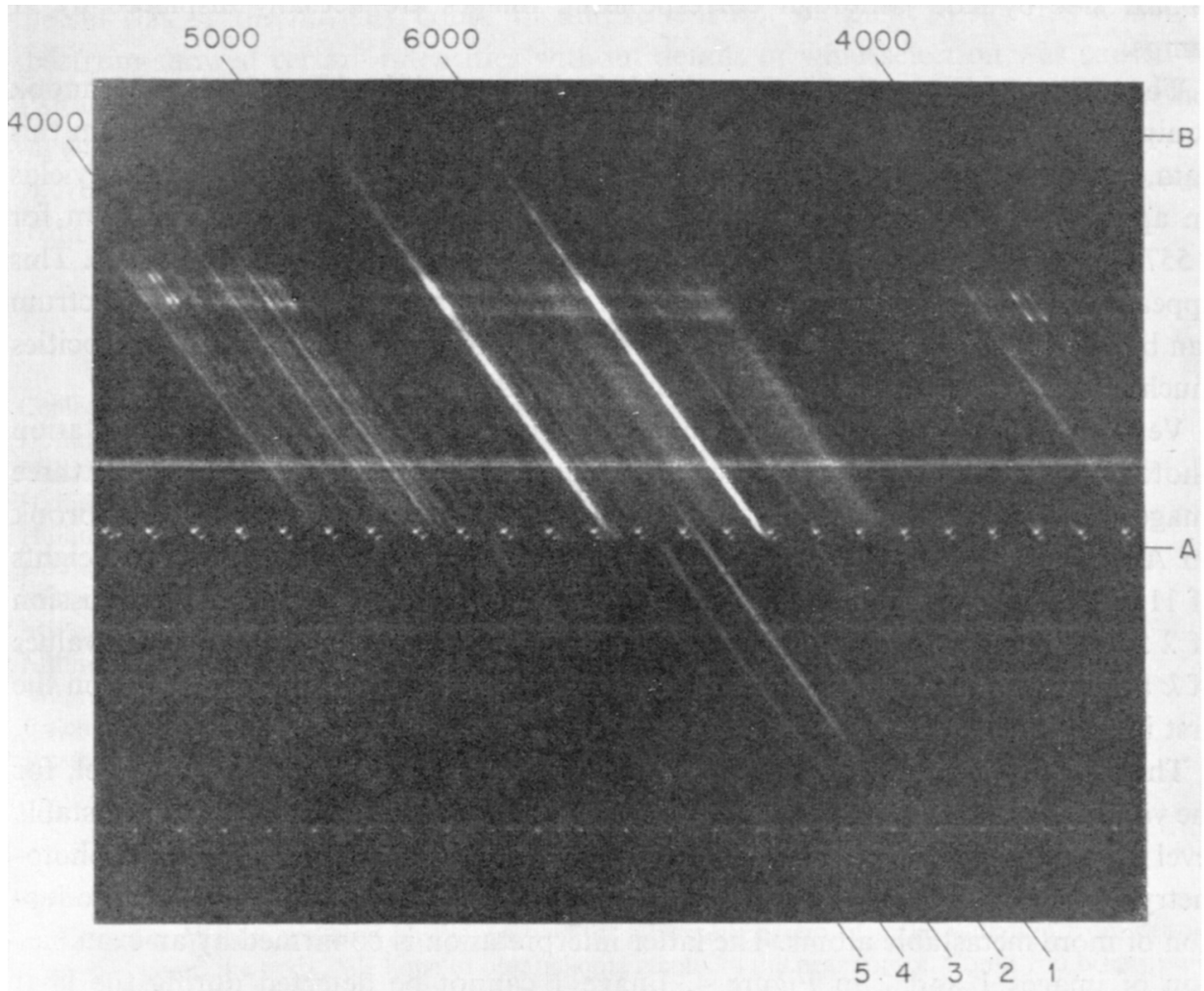

FIG. 4. Spectrum of a sporadic meteor photographed with camera 150 at Meanook. The meteor moved from bottom to top in the figure. The film was advanced abruptly when the meteor was at position $A$ and again at position $B$. Five consecutive images of $\lambda 5577$ were recorded as shown at the bottom. The wavelength scale at the top corresponds to the main meteor spectrum photographed between points $A$ and $B$. 
of a sporadic meteor photographed with camera 150 at Meanook on October 5, 1964, at $8^{\mathrm{h}} 48^{\mathrm{m}} 07^{\mathrm{s}} \mathrm{UT}$. Five consecutive images of $\lambda 5577$ were recorded, and these are numbered at the bottom of the figure. The film jumped when the meteor was at position $A$ and again at position $B$. Position $A$ happened to correspond closely to a major brightening of the meteor. Image 1 of $\lambda 5577$ was terminated when the film jumped at $A$, image 2 was recorded while the meteor traversed the distance $A$ to $B$, the meteor ended near the top of the figure shortly after the start of image 3 , and images 4 and 5 were recorded entirely after the end of the meteor. Image 1 is detectable as early as $0.3 \mathrm{sec}$ before the jump at $A$. Image 5 , which is extremely weak, was thus recorded between 1.5 and $1.9 \mathrm{sec}$ after the meteor was first detected. Image 3 of $\lambda 5577$ appears to be somewhat strengthened for a short distance, just before the jump at $A$, by overlap with the initial image of $\mathrm{MgI}, 2$, near $\lambda$ 5176. The two rows of dots near position $A$ are the zero-order images of $\alpha$ and $\beta$ Cass, which happen to appear side by side although corresponding images are actually displaced by 11 jumps.

The meteor of Figure 4 was also recorded with a normal spectrograph at Meanook equipped with a rotating shutter. Rigorous heights can not be determined from this data, but a study of the change of angular velocity on this second spectrogram yields an approximate radiant which is consistent with a height range of $115-97 \mathrm{~km}$ for $\lambda 5577,77 \mathrm{~km}$ for the bottom of the trail, and a meteoric velocity of $52 \mathrm{~km} / \mathrm{sec}$. This appears to be a normal height range for $\lambda 5577$ and the excitation of the spectrum can be reconciled with this velocity. The radiant is far above the ecliptic, so velocities much higher than $52 \mathrm{~km} / \mathrm{sec}$ can be eliminated from orbital considerations.

Very few of the meteors showing multiple images of the green line had two-station photographs suitable for detailed height calculations. A Perseid meteor showing three images of $\lambda 5577$ on camera 100 was photographed at both Meanook and Newbrook on August 5, 1964, at $7^{\mathrm{h}} 24^{\mathrm{m}} 04^{\mathrm{s}} \mathrm{UT}$. The green line was recorded between heights of 116 and $95 \mathrm{~km}$. Assuming an exponential decay of the form $e^{-k t}$ for the emission of $\lambda 5577$, visual photometry of the multiple images was employed to derive values of $k$ at different heights, allowing for the variable exposure of different points on the first image. The values of $k$ range from 0.4 at $116 \mathrm{~km}$ to 4.2 at $103 \mathrm{~km}$.

There is, however, evidence that an exponential decay is too simple a model, for the value of $k$ at $116 \mathrm{~km}$ implies a slower decay than the natural rate of the metastable level $(k=1 \cdot 0)$. This suggests either that there are considerable errors in the photometry or that in the initial stages the decay is counteracted by the continued production of more metastable atoms. The latter interpretation is confirmed by an examination of images 1 and 2 in Figure 4. Image 1 cannot be detected during the final 80 millisec before the jump at $A$, whereas image 2 in this region (near $105 \mathrm{~km}$ ) is some two magnitudes above the plate limit with an exposure of 400 millisec. These values do not fit a negative exponential but show that the peak instantaneous intensity of $\lambda 5577$ is not attained until some 50 or 100 millisec after the passage of the meteor. 
The mechanism of excitation to the metastable level is likely not a simple one and it appears to operate for these appreciable intervals.

The jumping-film spectra also show that the peak intensity of $\lambda 5577$ moves up the trail, from a height of about $100 \mathrm{~km}$ to $110 \mathrm{~km}$, during the decay period.

\section{Conclusions}

Meteor spectra suitable for detailed photometry are now being secured more commonly than in the past, although accurate calibration remains a problem in many cases. If this observational data is to yield quantitative results on meteor luminosity then it is essential to consider the detailed effects of an occulting shutter on any particular point on the trail where photometry is performed. Just as it is no longer sufficient to record the spectrum of a comet without specifying whether the slit was on the nucleus, coma, or tail, so it is not sufficient to say that a meteor spectrum showed certain intensities without details of what selection was caused by a shutter or, as also shown, by the location of the observer. We must decide whether we wish to consider the radiation coming from the immediate vicinity of the meteoroid or from some other specified location and time, and then proceed to derive the data from a suitable location in a suitable spectrogram.

\section{References}

Ceplecha, Z. (1966) Bull. astr. Inst. Csl., 17, 195.

Halliday, I. (1958a) Astrophys. J., 127, 245.

Halliday, I. (1958b) Astrophys. J., 128, 441.

Halliday, I. (1960) Astrophys. J., 131, 25.

Halliday, I. (1963) Smithson. Contr. Astrophys., 7, 161 ; Publ. Dom. Obs., Ottawa, $25,47$.

Halliday, I., Griffin, A. A. (1963) J. sci. Instruments, 40, 187; Publ. Dom. Obs., Ottawa, $25,39$.

Millman, P.M. (1950) Nature, 165, 1013.

Millman, P.M. (1956) Sky Telesc., 15, 445.

Millman, P. M. (1961) J. R. astr. Soc. Can., 55, 265.

Millman, P.M. (1963) J. R. astr. Soc. Can., 57, 89.

Rajchl, J. (1965) Bull. astr. Inst. Csl., 16, 282.

Russell, J.A. (1964) Meteoritics, 2, 117.

\section{DISCUSSION}

Ceplecha: We are working on the problem of a possible origin of wake radiation from droplets sprayed behind the body. We hope to obtain some results in the near future. Could you briefly repeat the reasons against the fragments or droplets being responsible for wake radiation in your spectrum?

Halliday: Because each position of the wake spectrum can be identified with the flare which produced it on the unocculted spectrogram, the retardation of the wake material can be measured during the interval between the flare and the beginning of the next segment of the trail. The retardation is much greater than $50 \%$ of the distance traversed by the meteor, which can only be caused by rapid initial deceleration as is expected for gas particles. 
McCrosky: The wake phenomena, if caused by fragments or droplets, is a strong function of velocity. Ceplecha's low-velocity iron can more reasonably be expected to produce 'droplet-wake' than Halliday's example at $60 \mathrm{~km} / \mathrm{sec}$.

Halliday: I agree that slow meteors will decelerate much more quickly than fast ones, but their retardation curve is still convex upwards, hence fragments or drops can never trail the meteor by as much as $50 \%$ of the distance travelled by the meteor since the particle was shed. 